\title{
Kernos
}

Revue internationale et pluridisciplinaire de religion

grecque antique

$30 \mid 2017$

Varia

\section{Divination and Human Nature. A Cognitive History of Intuition in Classical Antiquity}

\section{Kevin Bouillot}

\section{Q OpenEdition \\ Journals}

Édition électronique

URL : http://journals.openedition.org/kernos/2535

DOI : $10.4000 /$ kernos.2535

ISSN : 2034-7871

\section{Éditeur}

Centre international d'étude de la religion grecque antique

\section{Édition imprimée}

Date de publication : 1 octobre 2017

Pagination : 346-350

ISSN : 0776-3824

\section{Référence électronique}

Kevin Bouillot, "Divination and Human Nature. A Cognitive History of Intuition in Classical Antiquity », Kernos [En ligne], 30 | 2017, mis en ligne le 01 octobre 2017, consulté le 24 septembre 2020. URL http://journals.openedition.org/kernos/2535 ; DOI : https://doi.org/10.4000/kernos.2535

Ce document a été généré automatiquement le 24 septembre 2020.

Kernos 


\title{
Divination and Human Nature. A Cognitive History of Intuition in Classical Antiquity
}

\author{
Kevin Bouillot
}

\section{RÉFÉRENCE}

Peter T. STRUCK, Divination and Human Nature. A Cognitive History of Intuition in Classical Antiquity, Princeton/Oxford, Princeton University Press, 2016. 1 vol. $16 \times 24 \mathrm{~cm}, \mathrm{x}+288$ p. ISBN : 978-0-691-16939-2.

1 Alors que l'histoire de la divination fait, depuis les années 1970, un retour remarquable dans l'étude des religions anciennes, les sciences cognitives ont fait plus récemment leur entrée dans le champ des sciences de l'Antiquité. Peter Struck se propose de croiser la thématique divinatoire avec cette approche épistémologique nouvelle et d'interroger ainsi la définition même de la divination, tant chez les Anciens que chez les Modernes.

Constatant en introduction les biais initiaux de l'historiographie divinatoire récente ${ }^{1}$, puis les quelques angles morts laissés par les études majeures qui ont marqué les dernières décennies en la matière ${ }^{2}$, Peter Struck propose de se concentrer sur le «moment divinatoire » (et non sur son contexte) et ainsi d'interroger les raisons pour lesquelles les Anciens voyaient des signes ${ }^{3}$ dans ce que nous qualifions aujourd'hui de phénomènes naturels (ou humains) aléatoires (ou inconscients). Seuls les philosophes fournissent à une telle entreprise les matériaux nécessaires, et l'A. rappelle qu'une nette majorité d'entre eux croyait en la réalité de cette divination (à l'exception notable, mais minoritaire, d'Épicure, et peut-être de Cicéron, dont la position dans le De divinatione, évoquée d'ailleurs dans la monographie, reste ambigüe). En confrontant les différents systèmes théoriques élaborés par les principaux penseurs (et les principales écoles philosophiques), l'A. ambitionne de dépasser l'écueil de l'opposition entre 
rationalité (philosophique) et irrationalité (divinatoire), mais aussi l'habituelle distinction entre divination artificielle/déductive et divination naturelle/intuitive inscrite dans le marbre de l'historiographie par A.Bouché-Leclercq ${ }^{4}$. Il s'agit alors d'interroger les perceptions philosophiques de ce surplus knowledge qu'apportait la divination aux sociétés anciennes et dans lequel l'A. reconnaît ce que nos sociétés modernes appellent (depuis le Moyen Âge), «l'intuition », et que les sciences cognitives s'efforcent de circonscrire depuis leur émergence au milieu du siècle dernier. C'est donc d'une divination toute humaine que traite l'ouvrage, en appui sur les réflexions des philosophes anciens sur l'homme, sa nature et sa cognition.

Dans un premier chapitre consacré à la pensée de Platon, et reposant notamment (mais pas exclusivement) sur le Timée, P.S. ébauche une définition platonicienne de la divination centrée sur l'acquisition d'un savoir «non discursif », découlant de procédés cognitifs inconscients et qui procèdent des régions inférieures de l'âme, sièges des instincts et des pulsions naturelles de l'homme, mais aussi organes de réception des messages et images émanant du divin et de la partie supérieure, raisonnante et divine de l'âme.

Suivant la chronologie philosophique, l'A. interroge ensuite Aristote, chez qui l'essentiel de la réflexion divinatoire se concentre et se résume dans la question du rêve

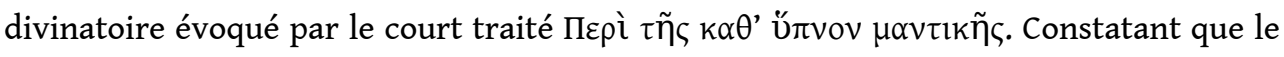
philosophe écarte les dieux du sujet, en faisant de cette divination par le rêve une propriété naturelle de l'âme humaine, l'A. relève une incohérence du discours aristotélicien qui qualifie ailleurs cette propriété de " démonique ». La résolution de ce puzzle et la clarification de la position d'Aristote passe alors par l'étude de ce que doit sa théorie du rêve mantique à la médecine ancienne (pour qui le rêve est d'abord un symptôme d'un état donné du corps), à la théorie aristotélicienne de la nature et du divin (le second ayant créé et mis en mouvement la première, qui agit ensuite selon son ontologie propre, qu'Aristote qualifie justement de démonique, mais aussi selon l'impulsion première donnée par le divin) et à l'explication par le philosophe de la

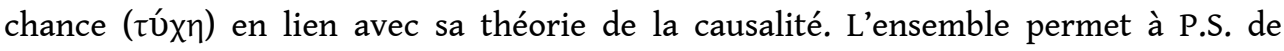
dégager une théorie aristotélicienne du rêve divinatoire mobilisant essentiellement la nature humaine (mise en lien avec celle du cosmos) et excluant l'intervention directe du divin.

Moins étudiés, moins bien documentés mais autrement plus prolixes sur les questions divinatoires, les stoïciens font l'objet du troisième chapitre, qui tire notamment parti de Posidonius et de ce qu'en rapporte Cicéron dans son De divinatione. Après avoir rappelé les piliers de la physique stoïcienne (autour des concepts de $\pi v \varepsilon \tilde{u} \mu \alpha$ et de "sympathie» notamment), l'A. constate que Posidonius prit ses distances avec la lecture stoïcienne d'une divination reposant sur la providence divine, pour développer une théorie déterministe (selon une mécanique cosmique complexe et reposant justement sur les $\pi v \varepsilon v ́ \mu \alpha \tau \alpha$ et leur sympathie), voire une théorie naturelle de la libération de l'âme humaine pendant le sommeil.

Enfin, le panorama de la chronologie philosophique s'achève par les néoplatoniciens et la figure centrale de Jamblique, auteur d'un discours divinatoire que P.S. qualifie de "restrictif et spécifique », malgré la filiation platonicienne de l'école tardive et le goût prononcé de Jamblique pour la question divinatoire. Après avoir cherché chez Platon, Virgile et Cicéron les prémices de cette pensée, l'A. constate la réorientation, par les néoplatoniciens, de la divination vers des sujets et surtout des vérités davantage 
cosmiques voire "théologiques $»^{5}$ que pragmatiques et contextuelles, comme l'illustrent notamment Porphyre et sa Philosophie tirée des oracles. Le traité de Jamblique longtemps intitulé Des mystères d'Égypte(aujourd'hui édité sous le titre de Réponse à Porphyre) achève enfin d'humaniser la divination en la scindant en deux branches. Tandis que la première procède toujours du divin, et même de sa présence réelle, en lien avec la théurgie que théorise justement le philosophe, l'autre branche, dans laquelle se retrouvent des méthodes tant naturelles qu'artificielles, est assimilée par Jamblique à une science conjecturale parmi d'autres, selon une conception matérielle voire matérialiste qui préfigure les critiques d'Origène ou d'Augustin d'Hippone.

7 Au terme du millénaire philosophique que balaye cette monographie, l'A. conclut donc à une remarquable convergence des grandes écoles philosophiques sur la question divinatoire, malgré des postulats cosmogoniques, théologiques, physiques, voire naturels radicalement différents. Tous tendraient à détacher la divination de son origine divine, pour l'arrimer ensuite aux propriétés de la nature (ou de l'âme, ou du $\pi v \varepsilon \tilde{V} \mu \alpha)$ humaine indépendante de toute intervention active du divin, et dans laquelle l'A. reconnaît une perception encore grossière et non formulée, mais émergente au fil des siècles et des écoles philosophiques, de ce que les sciences cognitives qualifient aujourd'hui d'intuition cognitive, c'est-à-dire la capacité à acquérir et/ou à exploiter des informations de manière non consciente et non discursive.

8 L'ouvrage et la démonstration ambitieuse sont servis par un plan simple et efficace, qui passe au crible cognitif les pensées philosophiques successives et s'efforce chaque fois d'en dégager les éléments confirmant l'hypothèse de départ, clairement explicitée d'emblée par l'A., et efficacement mise en pratique en conclusion, à travers la relecture de la séquence homérique du retour d'Ulysse et du massacre des prétendants, repensée comme témoignant des prémices de cette vision (encore divinatoire et divine) de la capacité humaine à «deviner». Les citations littéraires sont nombreuses, toujours accompagnées des traductions personnelles de l'A. et des références précises permettant de les resituer dans l'œuvre d'origine. Des schémas bienvenus viennent expliciter et synthétiser les constructions philosophiques les plus complexes, et l'A. n'oublie jamais qu'il ne s'adresse pas seulement à des spécialistes de philosophie antique. Son ouvrage jette une lumière nouvelle sur la pensée divinatoire ancienne et invite à dépasser certains écueils stériles (comme la question de la rationalité ou irrationalité de la divination) tout en réévaluant le prestige et l'importance de la divination (au moins dans la pensée philosophique).

L'ambition de l'A. d'appliquer ses conclusions non seulement au monde des philosophes anciens mais à l'ensemble des sociétés gréco-romaines interrogera davantage. Comment concilier ce constat d'une humanisation progressive de la divination avec les centaines de sanctuaires oraculaires du monde gréco-romain? Ceux-ci, jusqu'aux dernières heures du paganisme antique, pratiquèrent quotidiennement une divination qui, par définition, procédait du divin et de sa providence. La réalité quotidienne de la divination grecque ne semble pas avoir suivi la voie tracée par les philosophes antiques, et que dégage P.S., vers une expulsion des dieux du champ divinatoire. En ce sens, il est regrettable que soit absent du matériau utilisé par l'A. l'ensemble de la documentation épigraphique (en dehors du fameux oracle gravé à Oenoanda de Lycie ${ }^{6}$, érigé depuis un siècle en exemple et qui mériterait une relecture et une réinterprétation complètes, tant il est problématique et peu fiable), alors même que l'étude de la divination notamment oraculaire - est aujourd'hui renouvelée de fond en comble par la 
publication et l'étude de matériaux épigraphiques particulièrement riches, comme les lamelles de Dodone, pour n'en citer qu'un exemple ${ }^{7}$. Ces mêmes oracles élargissent d'ailleurs le fossé entre la divination des philosophes et celle des cités et des individus en conditionnant la réflexion d'un Plutarque, grand absent du tour d'horizon philosophique proposé par l'A. Pour ce prêtre de Delphes, qui fait si souvent la somme des écoles philosophiques dans ses dialogues, la divination n'est certainement pas affaire d'hommes seulement, mais procède et témoigne d'une providence divine et démonique dont il développe la théorie dans ses Dialogues pythiques notamment ${ }^{8}$. Plus que tout autre oracle, Delphes tenait également une place à part dans la réflexion divinatoire voire théologique de Platon et même de Jamblique (conjointement avec Claros et Didymes pour le second), place et particularité qui ne sont pas traitées par cet ouvrage. Au sein même du monde philosophique sur lequel se concentre P.S., l'unanimité à laquelle il conclut ne semble pouvoir se faire qu'en écartant certains théoriciens antiques. L'éviction des épicuriens, et autres sceptiques hostiles à la divination, laisse d'ailleurs de côté tout un pan de la réflexion divinatoire et providentielle (même s'il s'agit d'une réflexion a contrario), interdisant notamment une étude du second livre du De divinatione dans lequel Cicéron affirme précisément l'opposé de ce qu'il faisait dire à son frère dans le premier. Généraliser ce constat d'une dé-divinisation de la mantique à l'ensemble de la pensée philosophique grecque à partir d'une liste d'auteurs choisis semble donc pour le moins hâtif. Et étendre cette généralisation à l'ensemble de la pensée grecque l'est plus encore quand tant de données littéraires, épigraphiques et archéologiques ramènent sans cesse l'historien de la divination aux dieux, à leurs sanctuaires, à leurs cultes et à leurs logoi.

Peter Struck s'est donc attaqué à un pan majeur - et encore peu exploré - du renouveau historiographique des études divinatoires, celui de la philosophie. Il en dégage une tendance majeure, mais vraisemblablement pas unique, à détacher la divination du théisme qui l'a vue naître, pour l'ériger en vertu de l'âme ou de la nature humaine et, même si le risque de téléologisme est grand, ouvrir la voie à sa rationalisation par les sciences cognitives. L'ouvrage est donc à lire par quiconque s'intéresse à cette question et est prêt à entamer une remise en question des nombreuses certitudes acquises dans ce domaine, mais propose une autre généralisation qui ne rend pas justice à la richesse de la divination grecque.

\section{NOTES}

1.

Qui a longtemps été envisagée conjointement avec la magie et le domaine de l'irrationnel, et donc reléguée aux marges des sociétés gréco-romaines depuis E.R. DODDS, The Greeks and the irrational, Berkeley et al., 1951.

2. Notamment M.A. FLOWER, The Seer in Ancient Greece, Berkeley, 2008; K. BEERDEN, Worlds full of signs: ancient Greek divination in context, Londres, 2013; B. DIGNAS, R. PARKER, G. STROUMSA (éd.), Priests and Prophets among Pagans, Jews and Christians, Paris, 2013 ; E. EIDINow, Oracles, curses, and risk among the ancient Greeks, Oxford, 2007 ; S. GEORGOUDI, R. KOCH PIETTRE, Fr. SCHMIDT (dir.), La raison des 
signes. Présages, rites, destin dans les sociétés de la Méditerranée ancienne, Leiden, 2012 ; S.I. JOHNSTON, P. STRUCK (éd.), Mantikê. Studies in Ancient Divination, Leiden, 2005 ; M. KAJAVA (éd.), Studies in Ancient Oracles and Divination, Rome, 2013.

3. Tel que défini par GEORGOUDI - KOCH PIETTRE - SCHMIDT, ibid.

4. A. BOUCHÉ-LECLERCQ, Histoire de la divination dans l'Antiquité. Divination hellénique et divination italique, Paris, $1879-1882$.

5. Avec toute la prudence nécessaire pour appliquer ce terme aux oracles et à la divination depuis l'article fondateur d'A.D. NOCK, « Oracles théologiques », REA 30 (1928), p. 280-290.

6. La lecture actuelle s'appuyant encore essentiellement sur la théorie de Louis ROBERT, «Un oracle gravé à Oinoanda ", CRAI, 1971 (3), p. 597-619.

7. Le nouveau corpus disponible étant publié par Tsélikas (Sotiris), Tà $\chi \rho \eta \sigma \tau \eta ́ \rho l \alpha ~ \varepsilon \lambda \alpha ́ \alpha \sigma \mu \alpha \tau \alpha \tau \eta \varsigma$

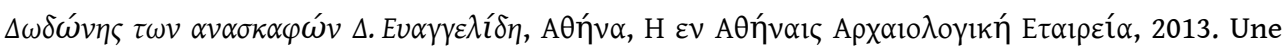
nouvelle édition de ces textes est en cours à Montréal (Dodona on line).

8. Voir la récente thèse de X. BRoullLETTE, La Philosophie delphique de Plutarque. L'itinéraire des Dialogues pythiques, Paris, 2014.

\section{AUTEURS}

\section{KEVIN BOUILLOT}

École Pratique des Hautes Études - PSL Paris / Université de Montréal 